\title{
CSF analysis differentiates multiple- system atrophy from idiopathic late-onset cerebellar ataxia
}

\author{
W.F. Abdo, MD; B.P.C. van de Warrenburg, MD, PhD; M. Munneke, PhD; W.J.A. van Geel; \\ B.R. Bloem, MD, PhD; H.P.H. Kremer, MD, PhD; and M.M. Verbeek, PhD, MSc
}

\begin{abstract}
Background: Differentiating idiopathic late-onset cerebellar ataxia (ILOCA) from ataxia due to the cerebellar subtype of multiple-system atrophy (MSA-C) can be difficult in the early stages of the disease Methods: The authors analyzed the levels of various CSF biomarkers in 27 patients with MSA-C and 18 patients with ILOCA and obtained cut-off points for each potential biomarker to differentiate MSA-C from ILOCA. Results: Increased levels of neurofilament light chain (NFL) and neurofilament heavy chain (NFHp35) and decreased levels of the neurotransmitter metabolites homovanillic acid (HVA), 5-hydroxyindoleaceticacid (5-HIAA), and 3-methoxy-4-hydroxyphenylethyleneglycol (MHPG) were observed in MSA-C compared with ILOCA patients. Receiver operating characteristic analysis showed high sensitivity and specificity levels for NFL, NFHp35, and MHPG analysis. At a cut-off of $24.4 \mathrm{ng} / \mathrm{L}$ for the NFL analysis, a sensitivity of $79 \%$ and a specificity of $94 \%$ were obtained for differentiating MSA-C from ILOCA. At a cut-off point for NFHp35 of $129.5 \mathrm{ng} / \mathrm{L}$, sensitivity was $87 \%$ and specificity $83 \%$. Analysis of MHPG levels (cut-off $42.5 \mathrm{nM}$ ) resulted in a sensitivity of $86 \%$ with a specificity of $75 \%$. A multivariate logistic regression model selected NFL, MHPG, and tau as independent predictive biomarkers that separated the MSA-C and ILOCA groups. Conclusions: Increased levels of neurofilament light chain and tau and decreased levels of 3-methoxy-4-hydroxyphenylethyleneglycol were associated with high accuracy levels in differentiating the cerebellar subtype of multiple-system atrophy from idiopathic late-onset cerebellar ataxia (LOCA). CSF analysis may thus serve as a useful tool in early diagnostic differentiation of LOCA.
\end{abstract}

NEUROLOGY 2006;67:474-479

Multiple-system atrophy (MSA) is a sporadic progressive neurodegenerative disorder of middle to late age, clinically characterized by a combination of parkinsonian, cerebellar, autonomic, and pyramidal signs. ${ }^{1-4}$ It is pathologically characterized by degeneration of various neuronal structures including the basal ganglia, brainstem, cerebellum, pyramidal tract, intermediolateral columns, and Onuf nucleus in the spinal cord. ${ }^{5,6}$ Cytoplasmic inclusions in oligodendroglial cells consisting of aggregated $\alpha$-synuclein are the neuropathologic hallmark of MSA. ${ }^{7,8}$ In contrast, idiopathic late-onset cerebellar ataxia (ILOCA) denotes a sporadic ataxia of unknown cause, clinically characterized by the slowly progressive pure cerebellar syndrome.

In most patients with the cerebellar form of MSA (MSA-C), additional noncerebellar symptoms may become apparent only as the disease progresses, making MSA-C difficult to distinguish from ILOCA in the earlier stages of the disease. However, differentiating MSA-C from ILOCA is important because of the prognostic differences between these two patient groups. Whereas ILOCA patients have a slowly progressive disease over many years, MSA-C patients have a more progressive course with earlier loss of mobility and a mean survival of 9 years. . $^{1,3,5,9,10}$

In search for diagnostic biomarkers, CSF analysis is potentially attractive because the CSF is contiguous with the extracellular fluid of brain structures. CSF biomarkers could thus reflect the differential pathologic changes that occur in the various neurodegenerative diseases. In this study, we analyzed multiple biomarkers in CSF from patients with MSA-C and ILOCA to investigate their ability to differentiate between these two forms of cerebellar degeneration.

Methods. Patients. We included all consecutive patients re-
ferred to the Department of Neurology of the Radboud University
Nijmegen Medical Centre between January 1996 and June 2003
for analysis of a sporadic late-onset cerebellar syndrome and who
underwent a lumbar puncture to exclude the possibility of an
infectious, immunologic, or paraneoplastic cause (table 1). Only
patients with MSA-C $(\mathrm{n}=27)$ or ILOCA $(\mathrm{n}=18)$ were included in
the statistical analysis, because these two disorders cause great
diagnostic uncertainty. Diagnostic evaluation included a detailed
disease history, a neurologic examination, blood pressure testing
while supine and standing, routine laboratory testing of blood and

From the Department of Neurology (W.F.A., B.P.C.v.d.W., M.M., B.R.B., H.P.H.K., M.M.V.) and Laboratory of Pediatrics and Neurology (W.J.A.v.G., M.M.V.), Radboud University Nijmegen Medical Centre, the Netherlands.

W.F.A was supported by a research grant of the Stichting Internationaal Parkinson Fonds.

Disclosure: The authors report no conflicts of interest.

Received September 19, 2005. Accepted in final form April 5, 2006.

Address correspondence and reprint requests to Dr. W.F. Abdo, Department of Neurology, Radboud University Nijmegen Medical Centre, P.O. Box 9101, 6500 HB Nijmegen, the Netherlands; e-mail: f.abdo@neuro.umcn.nl 
Table 1 Patient characteristics

\begin{tabular}{lccc}
\hline & MSA-C & ILOCA & $p$ Value \\
\hline No. of patients & 27 & 18 & \\
Disease severity & $1.6 \pm 0.58$ & $1.5 \pm 0.67$ & NS \\
Age, y & $61.1 \pm 7.7$ & $61.2 \pm 10.5$ & NS \\
Age at onset, y & $56.6 \pm 7.9$ & $53.8 \pm 10.8$ & NS \\
Disease duration at time of & $4.5 \pm 3.0$ & $7.3 \pm 4.4$ & 0.02 \\
$\quad$ lumbar puncture, y & & & \\
Clinical follow-up, y & $2.3 \pm 1.9$ & $2.5 \pm 2.7$ & NS \\
Disease duration at time of & $5.9 \pm 2.0$ & $8.4 \pm 4.2$ & 0.03 \\
$\quad$ last clinical visit, y & & & \\
\hline
\end{tabular}

Data are presented as means \pm SD. The $p$ value for differences using independent samples $t$ test with correction for unequal variances where applicable.

MSA-C = multiple-system atrophy dominated by cerebellar symptoms; ILOCA = idiopathic late-onset cerebellar ataxia.

urine, and a brain MRI or CT. In addition, all patients underwent extensive ancillary investigations that included metabolic screening (vitamin E, free thyroxine and thyroid-stimulating hormone, ceruloplasmin, $\alpha$-fetoprotein, very long chain fatty acids, lysosomal enzymes, urine organic acid, serum anti-gliadin IgG and IgM antibodies, and anti-endomysial antibodies; not all patients underwent all studies), iodobenzamide (IBZM-SPECT), neurophysiologic testing including anal sphincter electromyography (EMG) and peripheral nerve conduction and EMG studies, heart rate variability analysis, and genetic analysis of the various $S C A \mathrm{mu}-$ tations $(S C A 1 / 2 / 3 / 6 / 7 / 14)$ or the Friedreich ataxia gene (FRDA). In January 2005, two clinicians analyzed all the clinical patient records retrospectively while blinded to the CSF data and established consensus diagnosis for each case.

Disease severity was scored according to a previously described disease stages score, which was slightly modified (stages 1.5 and 2.5 were added). The following stages were defined: stage $0=$ no gait difficulties; stage $1=$ ataxia but walking independently; stage 1.5 = ataxia and intermittently dependent on walking aids stage $2=$ ataxia with permanent use of walking aids; stage $2.5=$ ataxia and intermittently dependent on wheelchair; stage $3=$ ataxia and permanently dependent on wheelchair; stage $4=$ death. $^{1}$

The diagnosis of MSA-C was made according to the consensus criteria proposed for probable and possible MSA. ${ }^{13}$ Patients with predominant cerebellar features were classified as MSA-C. Patients fulfilling the clinical criteria for MSA-P (clinically dominated by parkinsonism) were excluded from further analysis.

There are no widely used criteria to diagnose ILOCA. At our center, ILOCA is diagnosed according to the following stringent criteria: 1 ) age at onset $>30$ (as the MSA criteria state that an age at onset of $<30$ years is an exclusion criterion for MSA); 2) pure cerebellar syndrome without any noncerebellar symptoms including extensor plantar responses and sustained orthostatic hypotension; 3) absence of severe sensory loss or severe polyneuropathy on nerve conduction studies and EMG; 4) absence of anal sphincter EMG abnormalities suggestive of MSA. In all 10 ILOCA patients that had undergone anal sphincter EMG, it was found to be normal. 5) No mutation in the SCA1, 2, 3, 6, 7, and 14 genes or in the $F R D A$ gene. In the ILOCA group, none of the subjects who were tested for a possible hereditary cause had an FRDA gene expansion $(\mathrm{n}=12)$ or a mutation in one of the $S C A$ genes $(\mathrm{n}=18) .6$ ) Negative family history for an ataxia syndrome; 7) absence of a potential toxic cause that could explain the ataxia (alcoholism, anticonvulsant drugs); 8) a slowly progressive disease course; 9) normal (extensive) ancillary investigations including liver, kidney, thyroid, vitamin $\mathrm{B}_{1}, \mathrm{~B}_{6}, \mathrm{~B}_{12}$, vitamin $\mathrm{E}$, and folic acid, lactate, cholesterol, lysosomal enzymes, organic acid, ceruloplasmin, antigliadin or anti-endomysial antibodies, paraneoplastic antibodies, and Venereal Disease Research Laboratories (VDRL) test.

Patients with an age at onset of $<30$ without SCA or FRDA mutations and a negative familial history were classified as earlyonset cerebellar ataxia (EOCA). Patients were classified as autosomal dominant cerebellar ataxia (ADCA) if a mutation in one of the $S C A$ genes was found or if they had a family history suggesting autosomal dominant inheritance.

The source population included 79 subjects with ataxia who had undergone a lumbar puncture during the period 1996 through 2003. These patients carried the following diagnoses: EOCA $(\mathrm{n}=$ $10), \operatorname{ADCA}(\mathrm{n}=8)$, cerebellar syndromes due to various $(\mathrm{n}=16)$ causes such as an infectious or autoimmune-mediated cerebellar ataxia $(\mathrm{n}=4)$, paraneoplastic ataxia $(\mathrm{n}=1)$, alcoholic ataxia $(\mathrm{n}=$ 1 ), or patients with ataxia in whom a definite diagnosis could not be established owing to lack of data or unclear diagnosis $(n=10)$.

Forty-five patients were included in this study with possible $(\mathrm{n}=1)$, probable $(\mathrm{n}=23)$, or definite $(\mathrm{n}=3)$ MSA-C or with ILOCA ( $\mathrm{n}=18$ ) (table 1 ). Of the 27 MSA-C patients, 11 were deceased at the time of patient record review. In all three patients who underwent autopsy of the brain, the clinical diagnosis of MSA-C was confirmed. The mean survival in these 11 deceased patients was 8.0 years. Mean age at lumbar puncture, mean age at onset, and clinical follow-up duration did not differ between MSA-C and ILOCA patients. Mean disease duration at time of the lumbar puncture was significantly longer in the ILOCA patients.

For determination of reference values of the brain-specific proteins in CSF, we selected patients with ages between 40 and 75 who were referred to the Department of Neurology between 1998 and 2002 who underwent a lumbar puncture in a diagnostic workup but who, after extensive examination, turned out not to have a neurologic disease and had normal CSF cell count, hemoglobin, bilirubin, total protein, lactate, glucose, and no oligoclonal IgG bands. For determination of reference values of the neurotransmitter metabolites, we selected 26 patients with ages between 45 and 75 who underwent a lumbar puncture but did not have any disorder expected to affect neurotransmitter metabolism.

CSF biomarkers. Compared with ILOCA, there is a more extensive neurodegeneration in MSA-C, which could be reflected by altered concentrations of brain-specific proteins. Furthermore, the basal ganglia are clearly affected in MSA-C, which could result in lower monoamine metabolites in the CSF. The following potential biomarkers were therefore studied: neuron-specific enolase (NSE), S-100B glial protein, myelin basic protein (MBP), glial fibrillary acidic protein (GFAP), tau protein, neurofilament light chain (NFL) protein, phosphorylated neurofilament heavy chain (NFHp35) protein, the monoamines homovanillic acid (HVA) and 5-hydroxyindoleacetic acid (5-HIAA), and 3-methoxy-4hydroxyphenylethyleneglycol (MHPG).

Both NSE and S-100B concentrations in CSF were analyzed using an immunoluminometric assay (Byk Sangtec, Dietzenbach, Germany) by using the Liaison automated analyzer (Byk Sangtec). MBP concentrations in CSF were analyzed by using a commercial ELISA (DSL, Webster, TX). GFAP concentrations were analyzed by using a homemade sandwich ELISA. ${ }^{14,15}$ Tau concentrations in CSF were analyzed by using the Innotest hTau assay (Innogenetics, Gent, Belgium).

A previously described sandwich ELISA was used to determine NFL levels. ${ }^{16}$ Levels of NFHp35 were determined using a modified version of the sandwich ELISA. ${ }^{17}$ In summary, the microtiter plates were coated with mouse anti-phosphorylated NFHp35 antibodies (SMI35; Sternberger Monoclonals) and subsequently incubated with bovine NFHp35 standard (ICN, Burlingame, CA) or CSF samples, polyclonal rabbit anti-NFHp35 antibody (Affiniti Research Products, Exeter, UK), and with polyclonal peroxidaselabeled goat anti-rabbit antibodies (Jackson, Immunoresearch, Westgrove, PA). Tetramethyl-benzidin (TMB) was used as a substrate in the peroxidase reaction, and absorbance was read at 450 $\mathrm{nm}$. Tris-buffered saline ( $\mathrm{pH} 8.9)$ was used as washing and dilution buffer. The detection limit of the assay was calculated as the mean plus $3 \mathrm{SD}$ of the zero standard signal from 34 measurements and was determined to be $17 \mathrm{ng} / \mathrm{L}$. Mean recovery of the assay was $91.2 \%(\mathrm{n}=14)$. The intra-assay variation coefficient (VC) was $8.3 \%$ at a concentration of $71 \mathrm{ng} / \mathrm{L}(\mathrm{n}=12), 22.5 \%$ at a concentration of $38 \mathrm{ng} / \mathrm{L}(\mathrm{n}=12)$, and $2.8 \%$ at a concentration of $423 \mathrm{ng} / \mathrm{L}(\mathrm{n}=12)$. The interassay VC was $18 \%$ at a concentration of $35 \mathrm{ng} / \mathrm{L}(\mathrm{n}=18)$

HVA and 5-HIAA in CSF were measured according to previously described methods..$^{18}$ As the concentrations of HVA and 5 -HIAA vary in the different fractions of CSF, ${ }^{18}$ we always use the 9th to 11th millilter fraction of the obtained CSF. MHPG was measured by using the same method with some minor modifica- 
tions. ${ }^{18,19}$ The CSF data of the neurotransmitter metabolites were excluded from the statistical analysis in case patients or controls were taking antiparkinsonian or antidepressive medication at the time of the lumbar puncture because of their possible influence on these CSF levels. This was the case in three MSA-C and one ILOCA patients.

Statistical analysis. Differences between the MSA-C and ILOCA patients were analyzed using the independent samples $t$ test with correction for unequal variances where applicable. Spearman rank correlation test was used to test associations between quantitative variables. Receiver operator characteristic (ROC) analysis was used to evaluate the value of the individual biochemical variables and their optimal cut-off values with respect to the discrimination of MSA-C from ILOCA. Multivariate logistic regression with forward selection procedures was used to identify variables that contributed independently to discriminate MSA-C from ILOCA. Only variables with a $p$ value of $<0.10$ were entered in the multivariate analysis. In case of NFHp35, we also logarithmically transformed these data because of the non-Gaussian distribution. Graphpad Prism (San Diego, CA) and SPSS software were used for statistical analysis.

Results. At time of the lumbar puncture, the clinical picture of the MSA patients was as follows: In 8 patients the clinical picture was compatible with the criteria for probable MSA-C and in 10 with the criteria of possible MSA-C. ${ }^{13}$ In another nine patients the clinical picture at time of the lumbar puncture was that of an unknown cerebellar ataxia not compatible with the criteria of MSA. For the MSA patients the disease duration at time of the lumbar puncture was 4.5 years. These patients were followed for a mean period of 2.3 years. The mean disease duration at time of the last clinical picture was 5.9 years. In the ILOCA patients the mean disease duration at time of the lumbar puncture was 7.3 years; we followed these patients for a mean period of 2.5 years, and the mean disease duration at time of the last clinical follow-up was 8.4 years.

CSF biomarkers in MSA-C vs ILOCA. Levels of brainspecific proteins NFL and NFHp35 were significantly higher in MSA-C vs ILOCA patients (figure, A and B; table 2). Tau levels were also increased in MSA-C but showed only a trend toward significance. Furthermore, levels of all neurotransmitter metabolites were significantly decreased in the MSA-C group compared with the ILOCA group, with MHPG showing the most significant difference (figure, C). We calculated optimal cut-off values and accompanying sensitivity and specificity values for the CSF biomarkers with a difference $(p<0.05)$ between MSA-C and ILOCA (table 3 ). In addition, we calculated the likelihood ratio and the area under the ROC curve. NFL analysis resulted in the largest area under ROC curve with the best accuracy to differentiate between MSA-C and ILOCA (table 3).

Studies addressing the relative frequencies of the various sporadic cerebellar ataxias are rare. In one study, $32 \%$ of patients with a sporadic cerebellar ataxia could be classified as ILOCA. ${ }^{20}$ Another study diagnosed MSA-C in $29 \%$ of patients presenting with a sporadic ataxia. ${ }^{1}$ So, a rough assumption would be that in the group of sporadic cerebellar ataxias, MSA-C would account for approximately 30\%, ILOCA for 30\%, and other diagnoses for the remaining $40 \%$. The proportion of MSA-C patients to ILOCA patients in this study was $3: 2$. Although this is slightly different from the 1:1 ratio extracted from the literature, we calculated positive and negative predictive values using this $3: 2$ ratio. The calculated positive (PPV) and negative (NPV) predictive values for the distinction between MSA-C and ILOCA were as follows: for NFL, the PPV was $95 \%$ with a
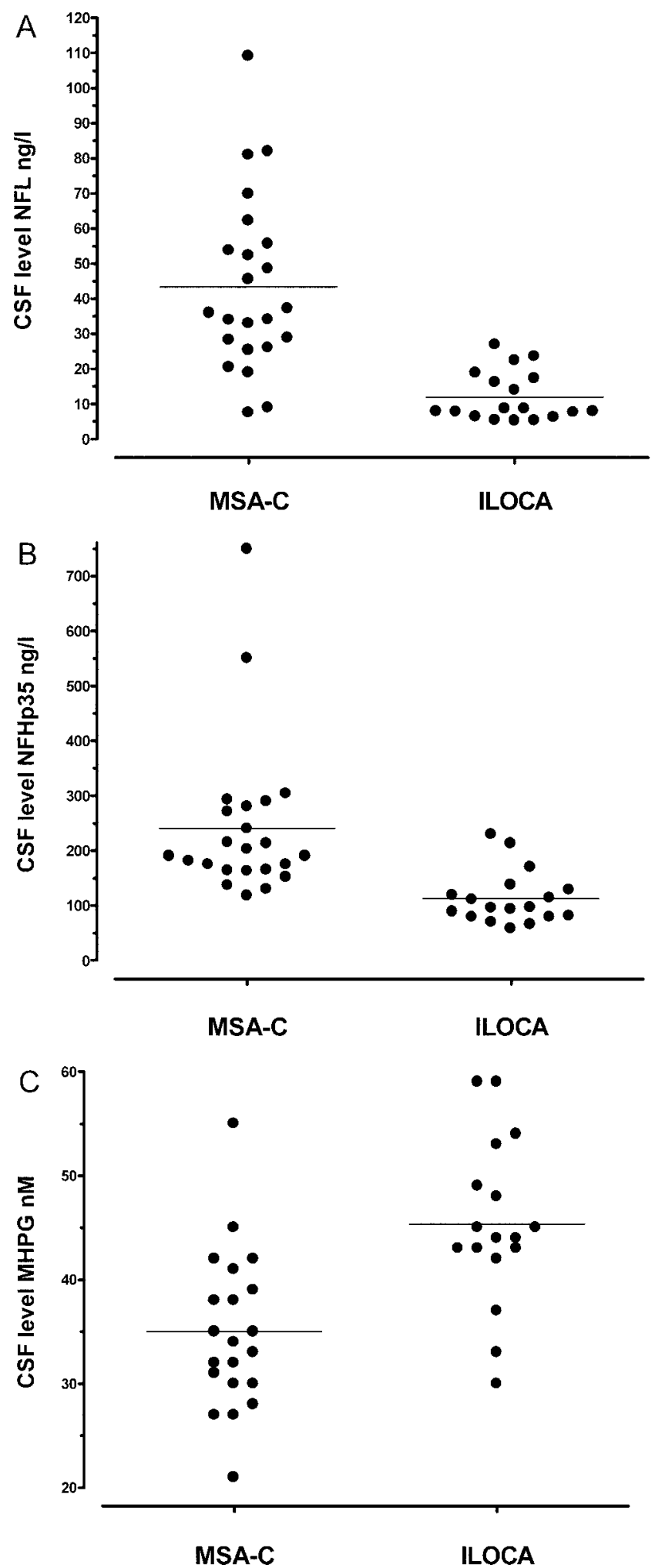

Figure. CSF levels of neurofilament light chain (A), neurofilament heavy chain p35 (B), and 3-methoxy-4hydroxyphenylethyleneglycol $(C)$ in the cerebellar form of multiple-system atrophy and idiopathic late-onset cerebellar ataxia groups. Horizontal lines indicate mean levels.

NPV of $76 \%$; for NFHp35, the PPV was $85 \%$ and the NPV was $87 \%$; and for the MHPG analysis, the PPV was $83 \%$ with a NPV of $80 \%$.

We performed multivariate logistic regression to iden- 


\begin{tabular}{lccc}
\hline & MSA-C & ILOCA & Controls \\
\hline Neurotransmitter metabolites & & & \\
HVA, nM & $135 \pm 73(22)$ & $199 \pm 59(14)$ & $228 \pm 128(31)$ \\
5-HIAA, nM & $68 \pm 33(22)$ & $100 \pm 30(14)$ & $126 \pm 55(31)$ \\
MHPG, nM & $35.8 \pm 8.3(22)$ & $44.9 \pm 8.0(16)$ & $47 \pm 12(28)$ \\
Brain-specific proteins & & & 0.01 \\
NFL, ng/L & $42 \pm 25(24)$ & $11.7 \pm 7(17)$ & $11.3 \pm 15(36)$ \\
NFHp35, ng/L & $234 \pm 144(24)$ & $115 \pm 49(17)$ & $83 \pm 64(33)$ \\
NSE, $\mu \mathrm{g} / \mathrm{L}$ & $9.3 \pm 4.0(25)$ & $9.5 \pm 2.7(18)$ & $8.8 \pm 4(68$ \\
MBP, $\mu \mathrm{g} / \mathrm{L}$ & $0.9 \pm 0.37(22)$ & $0.81 \pm 0.40(17)$ & $0.42 \pm 0.34(59)$ \\
Tau, ng/L & $309 \pm 117(25)$ & $237 \pm 132(18)$ & $171 \pm 99(79)$ \\
GFAP, $\mu \mathrm{g} / \mathrm{L}$ & $2.2 \pm 1.2(21)$ & $2.0 \pm 1.3(18)$ & $1.0 \pm 1.1(67)$ \\
S-100B, $\mu \mathrm{g} / \mathrm{L}$ & $2.7 \pm 0.9(24)$ & $2.3 \pm 0.9(18)$ & 0.0001 \\
A $\beta_{42}, \mathrm{Ng} / \mathrm{L}$ & $755 \pm 241(25)$ & $768 \pm 175(18)$ & $2.4 \pm 0.62(68)$ \\
\hline
\end{tabular}

Values are means $\pm \mathrm{SD}(\mathrm{n})$. The $p$ value for statistical differences between MSA-C and ILOCA using the independent samples $t$ test with correction for unequal variances where applicable.

MSA-C = cerebellar form of multiple-system atrophy; ILOCA = idiopathic late-onset cerebellar ataxia; HVA = homovanillic acid; 5-HIAA = 5-hydroxyindoleacetic acid; MHPG = 3-methoxy-4-hydroxyphenylethyleneglycol; NFL = neurofilament light chain; NFHp35 = phosphorylated neurofilament heavy chain; NSE $=$ neuron-specific enolase; $\mathrm{MBP}=$ myelin basic protein; GFAP $=$ glial fibrillary acidic protein; $\mathrm{A} \beta_{42}=$ amyloid $\beta$-protein.

tify CSF variables that contributed independently to the differentiation between MSA-C and ILOCA. All CSF parameters with a $p$ value of $<0.10$ were entered in this multivariate analysis. With use of this method, NFL, MHPG, and tau were selected according to the following model: $X=162-6.4 * \mathrm{NFL}+2.3 * \mathrm{MHPG}-0.5 *$ tau. With use of this mathematical model, the MSA and ILOCA groups were perfectly (100\%) separated. Because two MSA-C patients had high NFHp35 levels (figure, B), we logarithmically transformed the NFHp35 data to obtain a Gaussian distribution and performed an alternative multivariate logistic regression with substitution of NFHp35 levels by the logarithmically transformed data. This did not alter the outcome of the multivariate analysis.

In MSA-C, all three measured neurotransmitter metabolites correlated well with each other, in particular 5-HIAA with HVA $(r=0.85 ; p<0.0001)$. Furthermore, NFL was

Table 3 Optimal cut-off values of the most significant CSF variables between MSA-C and ILOCA

\begin{tabular}{lccccc}
\hline \multirow{2}{*}{$\begin{array}{l}\text { CSF } \\
\text { biomarker }\end{array}$} & \multicolumn{3}{c}{ Cut-off } & \multicolumn{3}{c}{ Sensitivity, } & \multicolumn{3}{c}{$\begin{array}{c}\text { Specificity, } \\
\text { value }\end{array}$} & $\%$ & & $\%$ & ratio & $\begin{array}{c}\text { ROC AUC } \\
(95 \% \text { CI })\end{array}$ \\
\hline NFL, ng/L & $>24.4$ & 79 & 94 & 13.5 & $0.93(0.86-1.0)$ \\
NFHp35, ng/L & $>145$ & 87 & 83 & 6.4 & $0.87(0.75-0.99)$ \\
MHPG, nM & $<42.5$ & 86 & 75 & 3.5 & $0.81(0.66-0.95)$ \\
HVA, nM & $<64$ & 71 & 68 & 2.4 & $0.76(0.60-0.91)$ \\
5-HIAA, nM & $<63.5$ & 59 & 100 & - & $0.78(0.63-0.93)$ \\
\hline
\end{tabular}

Optimal cut-off was calculated using univariate logistic regression with ROC analysis. Sensitivity for the diagnosis of MSA is calculated by assignment of MSA-C as patients and ILOCA as controls.

ROC AUC = receiver operating characteristic area under curve; MSA-C = cerebellar form of multiple-system atrophy; ILOCA = idiopathic late-onset cerebellar ataxia; NFL = neurofilament light chain; NFHp35 = phosphorylated neurofilament heavy chain; HVA = homovanillic acid; 5-HIAA = 5-hydroxyindoleacetic acid; MHPG = 3-methoxy-4hydroxyphenylethyleneglycol. correlated with MHPG $(r=-0.51 ; p=0.03)$ and NFHp35 $(r=0.476 ; p=0.02)$. In ILOCA, MHPG correlated with age $(r=0.68 ; p=0.004)$ and age at onset $(r=0.65, p=$ $0.006)$. Tau levels correlated with age $(r=0.60, p=0.009)$ and age at onset $(r=0.64, p=0.005)$, NFL $(r=-0.66$, $p=0.004)$, NSE $(r=0.76, p=0.0002)$, and MHPG $(r=$ $0.51, p=0.05)$. Also, GFAP correlated with $\mathrm{NFH}(r=0.53$, $p=0.03)$, and HVA and HIAA levels were correlated ( $r=$ $0.62 ; p=0.02)$.

Discussion. We observed clear differences in the levels of various CSF biomarkers between MSA-C and ILOCA, which suggests that CSF analysis could aid in the differential diagnosis of sporadic late-onset ataxias. First, we found that the levels of NFL and NFHp35, but not the other brain-specific proteins, were increased in MSA-C compared with ILOCA. Second, the levels of the neurotransmitter metabolites were decreased in MSA-C. Finally, multivariate logistic regression analysis showed that a combination of NFL, tau, and MHPG could accurately differentiate MSA-C from ILOCA.

Levels of NFL and NFHp35 were significantly increased in the MSA patients and resulted in high sensitivity and specificity levels to differentiate MSA-C from ILOCA patients. Both animal and human studies have shown that NF proteins play a major role in the axonal environment. NF proteins are highly phosphorylated neuronal cytoskeleton proteins composed of three subunits defined by their molecular weight on sodium dodecyl sulfate gel electrophoresis: $68 \mathrm{kd}$ (NFL), $160 \mathrm{kd}$ (NF medium chain), and $200 \mathrm{kd}$ (NFHp35). NFL is essential for the assembly of the NF proteins and forms the backbone of the neurofilament structures. NF proteins are essential for maintaining neuronal cytoskeletal 
plasticity by influencing axonal caliber and axonal transport. ${ }^{21}$ In a mouse model with MSA-like neurodegeneration and pathology, phosphorylated $\mathrm{NFH}$ redistributed to the neuronal perikarya as a consequence of axonal injury. ${ }^{22}$ Previous studies found elevated CSF levels of NFL in neurologic diseases associated with axonal injury such as in multiple sclerosis. ${ }^{23}$ In a small study of patients with ALS, higher NFL levels were present in patients with upper motor neuron involvement relative to those with only lower motor neuron involvement. ${ }^{24}$ Furthermore, higher levels of NFL have been reported in MSA-P relative to Parkinson disease. ${ }^{25,26}$ Therefore, NF proteins could be regarded as biomarkers of degeneration of (especially large myelinated) axons. Axonal degeneration within, for example, the pyramidal tracts could thus result in higher NF protein levels in the CSF. In this study, higher levels of NFL and NFHp35 were observed in MSA-C, possibly reflecting the more widespread neuronal degeneration that leads to secondary axonal decline in MSA-C. Furthermore, involvement of the pyramidal tract, which is an established feature of MSA-C pathology, could partially have contributed to the higher levels of NFL and NFHp35 in MSA-C.

CSF levels of tau protein tended to be increased in the MSA-C group. Tau is a microtubule-associated protein mainly localized in neuronal axons where it stabilizes the microtubules that are essential for axonal transport and maintains the function of neuronal cytoskeleton. Similar to NF proteins, widespread axonal degeneration in MSA-C could have resulted in the higher CSF tau levels. The levels of other brain-specific proteins did not differ significantly between both groups, indicating that the axonal proteins (NF and tau) are a more sensitive marker for differences in neuronal degeneration with secondary axonal damage.

We also found that the mean levels of neurotransmitter metabolites were lower in MSA-C compared with ILOCA patients. These findings are consistent with other publications in MSA patients and correspond to the degeneration of the basal ganglia and neurotransmitter producing nuclei in the brainstem. ${ }^{12,27}$ CSF MHPG was the most discriminative neurotransmitter metabolite. MHPG is a noradrenergic neurotransmitter metabolite, and decreased CSF concentrations in MSA may reflect noradrenergic depletion caused by loss of catecholaminergic neurons in the locus ceruleus and ventrolateral medulla in MSA. ${ }^{28,29}$ Features of autonomic dysfunction are observed in almost all MSA patients and correlate with lower CSF MHPG levels. ${ }^{19,29-32}$ The observed correlations in MSA-C between the various neurotransmitter metabolites are in agreement with earlier publications in MSA and Parkinson disease, suggesting that when degeneration of the (monoamine-producing) basal ganglia develops, this is not confined to one localized neuronal population but simultaneously affects multiple areas in the basal ganglia. ${ }^{19}$ These correlations were also partially observed in ILOCA, where HVA and HIAA were correlated. In MSA-C, levels of MHPG tended to be decreased when levels of NFL were higher, suggesting that in MSA ongoing axonal degeneration (reflected by the higher NFL levels) parallels the degeneration of noradrenaline-producing nervous structures.

Our retrospective study has some potential shortcomings. First, the number of patients in the ILOCA group was relatively small. However, because of our stringent inclusion criteria for ILOCA, these patients present a homogeneous group. Consequently, despite the relative small numbers, we found clear differences in CSF levels. Second, logistic regression analysis was perhaps suboptimal owing to some missing data of the CSF parameters. Third, we cannot exclude some form of selection bias. It is standard procedure at our center to perform a lumbar puncture in patients who present with a sporadic LOCA of unknown etiology, to exclude the possibility of an infectious, immunologic, or paraneoplastic cause. However, occasional patients did not undergo a lumbar puncture, thereby introducing a selection bias. Finally, the disease duration at the time of the lumbar puncture was significantly longer in the ILOCA group. However, disease duration was not correlated with the different CSF parameters in both groups, meaning that this difference is not likely to explain all observed differences. The fact that we did not observe a correlation between CSF biomarkers and disease duration might suggest that this assessment could be useful early in the disease course. Furthermore, all eight patients who had CSF analysis within 2 years of disease course had a perfect or near perfect CSF profile compatible with their eventual diagnosis. However, a prospective study is needed to establish the value of early CSF biomarker analysis.

\section{Acknowledgment}

The authors thank the technicians of the Laboratory of Pediatrics and Neurology for CSF analysis.

\section{References}

1. Abele M, Burk K, Schols L, et al. The aetiology of sporadic adult-onset ataxia. Brain 2002;125:961-968.

2. Schols L, Szymanski S, Peters S, et al. Genetic background of apparently idiopathic sporadic cerebellar ataxia. Hum Genet 2000;107:132137.

3. Gilman S, Little R, Johanns J, et al. Evolution of sporadic olivopontocerebellar atrophy into multiple system atrophy. Neurology 2000;55: 527-532.

4. Quinn N. Multiple system atrophy-the nature of the beast. J Neurol Neurosurg Psychiatry 1989;suppl:78-89.

5. Wenning GK, Tison F, Ben Shlomo Y, Daniel SE, Quinn NP. Multiple system atrophy: a review of 203 pathologically proven cases. Mov Disord 1997;12:133-147.

6. Ozawa T, Paviour D, Quinn NP, et al. The spectrum of pathological involvement of the striatonigral and olivopontocerebellar systems in multiple system atrophy: clinicopathological correlations. Brain 2004; 127:2657-2671.

7. Papp MI, Kahn JE, Lantos PL. Glial cytoplasmic inclusions in the CNS of patients with multiple system atrophy (striatonigral degeneration, olivopontocerebellar atrophy and Shy-Drager syndrome). J Neurol Sci 1989;94:79-100

8. Goedert M. Alpha-synuclein and neurodegenerative diseases. Nat Rev Neurosci 2001;2:492-501.

9. Watanabe H, Saito $\mathrm{Y}$, Terao $\mathrm{S}$, et al. Progression and prognosis in multiple system atrophy: an analysis of 230 Japanese patients. Brain 2002;125:1070-1083. 
10. Klockgether T, Schroth G, Diener HC, Dichgans J. Idiopathic cerebellar-ataxia of late onset-natural-history and MRI morphology. J Neurol Neurosurg Psychiatry 1990;53:297-305.

11. Kaakkola S, Tuomainen P, Mannisto PT, Palo J. Biogenic-amine metabolites in the CSF of patients with late-onset and alcoholic ataxias. Acta Neurol Scand 1993;87:309-311.

12. Higgins JJ, Harvey White JD, Nee LE, Colli MJ, Grossi TA, Kopin IJ. Brain MRI, lumbar CSF monoamine concentrations, and clinical descriptors of patients with spinocerebellar ataxia mutations. J Neurol Neurosurg Psychiatry 1996;61:591-595.

13. Litvan I, Bhatia KP, Burn DJ, et al. Movement Disorders Society Scientific Issues Committee report: SIC Task Force appraisal of clinical diagnostic criteria for parkinsonian disorders. Mov Disord 2003;18:467486.

14. Van Geel WJ, de Reus HP, Nijzing H, Verbeek MM, Vos PE, Lamers KJ. Measurement of glial fibrillary acidic protein in blood: an analytical method. Clin Chim Acta 2002;326:151-154.

15. Herrmann M, Vos P, Wunderlich MT, de Bruijn CH, Lamers KJ. Release of glial tissue-specific proteins after acute stroke: a comparative analysis of serum concentrations of protein S-100B and glial fibrillary acidic protein. Stroke 2000;31:2670-2677.

16. Van Geel WJ, Rosengren LE, Verbeek MM. An enzyme immunoassay to quantify neurofilament light chain in cerebrospinal fluid. $\mathrm{J}$ Immunol Methods 2005;296:179-185.

17. Petzold A, Keir G, Green AJ, Giovannoni G, Thompson EJ. A specific ELISA for measuring neurofilament heavy chain phosphoforms. J Immunol Methods 2003;278:179-190.

18. Brautigam C, Wevers RA, Jansen RJ, et al. Biochemical hallmarks of tyrosine hydroxylase deficiency. Clin Chem 1998;44:1897-1904.

19. Abdo WF, de Jong D, Hendriks JCM, et al. Cerebrospinal fluid analysis differentiates multiple system atrophy from Parkinson's disease. Mov Disord 2004;19:571-579.

20. Ormerod IE, Harding AE, Miller DH, et al. Magnetic resonance imaging in degenerative ataxic disorders. J Neurol Neurosurg Psychiatry 1994;57:51-57.

21. Fuchs E, Cleveland DW. A structural scaffolding of intermediate filaments in health and disease. Science 1998;279:514-519.
22. Yazawa I, Giasson BI, Sasaki R, et al. Mouse model of multiple system atrophy alpha-synuclein expression in oligodendrocytes causes glial and neuronal degeneration. Neuron 2005;45:847-859.

23. Lycke JN, Karlsson JE, Andersen O, Rosengren LE. Neurofilament protein in cerebrospinal fluid: a potential marker of activity in multiple sclerosis. J Neurol Neurosurg Psychiatry 1998;64:402-404.

24. Rosengren LE, Karlsson JE, Karlsson JO, Persson LI, Wikkelso C. Patients with amyotrophic lateral sclerosis and other neurodegenerative diseases have increased levels of neurofilament protein in CSF. J Neurochem 1996;67:2013-2018.

25. Holmberg B, Johnels B, Ingvarsson P, Eriksson B, Rosengren L. CSFneurofilament and levodopa test combined with discriminant analysis may contribute to the differential diagnosis of parkinsonian syndromes. Parkinsonism Relat Disord 2001;8:23-31.

26. Holmberg B, Rosengren L, Karlsson JE, Johnels B. Increased cerebrospinal fluid levels of neurofilament protein in progressive supranuclear palsy and multiple-system atrophy compared with Parkinson's disease. Mov Disord 1998;13:70-77.

27. Botez MI, Young SN. Biogenic amine metabolites and thiamine in cerebrospinal fluid in heredo-degenerative ataxias. Can J Neurol Sci 2001; 28:134-140.

28. Benarroch EE, Smithson IL, Low PA, Parisi JE. Depletion of catecholaminergic neurons of the rostral ventrolateral medulla in multiple systems atrophy with autonomic failure. Ann Neurol 1998;43:156-163.

29. Mathias CJ, Williams AC. The Shy-Drager syndrome (and multiple system atrophy). In: Calne DB, ed. Neurodegenerative diseases. Philadelphia: WB Saunders, 1994:743-767.

30. Polinsky RJ, Jimerson DC, Kopin IJ. Chronic autonomic failure: CSF and plasma 3-methoxy-4-hydroxyphenylglycol. Neurology 1984;34:979_ 983.

31. Wenning GK, Ben SY, Magalhaes M, Daniel SE, Quinn NP. Clinical features and natural history of multiple system atrophy. An analysis of 100 cases. Brain 1994;117:835-845

32. Watanabe H, Saito Y, Terao S, et al. Progression and prognosis in multiple system atrophy: an analysis of 230 Japanese patients. Brain 2002;125:1070-1083.

\section{P WWW.NEUROLOGY.ORG OFFERS IMPORTANT INFORMATION TO PATIENTS AND THEIR FAMILIES}

The Neurology Patient Page provides:

- a critical review of ground-breaking discoveries in neurologic research that are written especially for patients and their families

— up-to-date patient information about many neurologic diseases

— links to additional information resources for neurologic patients.

All Neurology Patient Page articles can be easily downloaded and printed, and may be reproduced to distribute for educational purposes. Click on the Patient Page icon on the home page (www.neurology.org) for a complete index of Patient Pages. 